\title{
Effect of BMPs and Wnt3a co-expression on the osteogenetic capacity of osteoblasts
}

\author{
WENDONG RUAN, YUAN XUE, YAQI ZONG and CHAO SUN \\ Department of Orthopaedics, The General Hospital of Tianjin Medical University, Tianjin 300052, P.R. China
}

Received June 26, 2015; Accepted June 30, 2016

DOI: $10.3892 / \mathrm{mmr} .2016 .5734$

\begin{abstract}
In the present study, third-generation autologous-inactivated bone morphogenic protein 2 (BMP2), BMP4, BMP6, BMP7, BMP9 and Wnt3a lentiviral vectors were constructed and integrated into the genome of MC3T3-E1 murine mesenchymal stem cells (MMSCs) to produce osteoinductive factor gene-modified MMSCs. The transfection efficiency of each osteoinductive factor was then determined by detecting the expression levels of runt related transcription factor 2 (Runx2) mRNA. The cotransfection with combinations of two lentiviruses was performed, and the expression levels of bone $\gamma$-carboxyglutamate protein and alkaline phosphatase in the MC3T3-E1 cell culture supernatant were detected. The expression level of Runx 2 mRNA was detected by reverse transcription-polymerase chain reaction, and western blotting was performed to detect the protein expression levels of BMP2, BMP4, BMP6, BMP7, BMP9 and Wnt3a. The results demonstrated that the recombinant lentiviruses were successfully transfected into MC3T3-E1 cells. The relative expression levels of Runx2 mRNA were greatest in the BMP2 group, sequentially followed by the BMP4, BMP9, BMP7, Wnt3a and BMP6 groups. The results of cotransfection of MC3T3-E1 cells (a total of 8 groups) demonstrated that BMP-2 and BMP-7 exhibited the highest cotransfection efficiency. Western blot analysis demonstrated that following BMP2 and BMP7 cotransfection of MC3T3-E1 cells, the protein expression levels of BMP2, BMP4, BMP6, BMP7, BMP9 and Wnt3a were increased compared with control cells. In conclusion, the third-generation lentiviral vectors effectively improved the osteogenic efficiencies of MC3T3-E1 cells, which provided an important theoretical basis and therapeutic strategy for bone reconstruction and tissue engineering.
\end{abstract}

Correspondence to: Professor Yuan Xue, Department of Orthopaedics, The General Hospital of Tianjin Medical University, 154 Anshan Road, Tianjin 300052, P.R. China

E-mail: yuanxuecn@126.com

Key words: bone regeneration, bone morphogenetic protein, wnt3a, lentiviral vector, MC3T3-E1 cells

\section{Introduction}

Bone regeneration is an important step in treating bone defects, fractured nonunion and osteoporosis. Previously, stem cells, including bone marrow-derived mesenchymal stem cells (MSCs) and others, have been used to solve the problem of obtaining seed cells for artificial bone tissue engineering $(1,2)$. An effective way to promote the osteogenic differentiation of seed cells was to use gene therapy techniques to transfect osteoinductive regulatory factors into the seed cells, and to effectively express the regulatory factors in the seed cells, thus contributing to the osteogenic differentiation of seed cells $(3,4)$.

Currently, there are various osteoinductive regulator types, among which the bone morphogenetic proteins (BMPs) have previously been heavily investigated. BMPs are part of the TGF- $\beta$ superfamily, which includes 43 members. It was previously demonstrated that BMPs could induce mesenchymal cells to irreversibly differentiate into cartilages and bone cells in vivo (5-7). Additionally, the wingless-type mouse mammary tumor virus integration site family is a class of Wnt gene-encoded secretory glycoproteins. The highly conserved Wnt signaling pathway is important in numerous species, it controls early embryonic development and regulates cell proliferation $(8,9)$. Selecting the ideal carrier, so that the target gene could be specifically, controllably and efficiently expressed, is crucial for successful gene therapy. The lentiviral vector system produces effectively expressed osteoinductive factors in the seed cells of artificial bone of bone tissue engineering, leading to high and prolonged expression of osteoinductive factors (10).

The current study was approved by was approved by the Ethics Committee of the General Hospital of Tianjin Medical University and aimed to construct a third-generation of autologous inactivated BMP2, BMP4, BMP6, BMP7, BMP9 and Wnt3a lentiviral vectors, and integrate them into the genome of MC3T3-E1 murine MSCs (MMSCs) to produce osteoinductive factor gene-modified MMSCs, and thus, determine effective ways to further enhance the osteogenic differentiation ability of the cells.

\section{Materials and methods}

Construction of pELNS-BMPs and pELNS-Wnt3a vectors. A cDNA library (Wuhan Genesil Biotechnology Co., Ltd., Wuhan, China) was used as the template, and polymerase 
chain reaction (PCR) was performed to amplify the coding sequences of BMP2, BMP4, BMP6, BMP7, BMP9 and Wnt3a, according to the method of the previously reported method (11). The gene sequencing results were: Mouse BMP2 (NM_007553.2), BMP4 (NM_007554.2), BMP6 (NM_007556.2), BMP7 (NM_007557.2), BMP9 (NM_019506.4), Wnt3a (NM_009522.2). The upstream PCR primer introduced an Nhe I restriction enzyme site and the downstream introduced a $\mathrm{Sal}$ I restriction enzyme site to the amplified sequences. The primer sequences used are presented in Table I.

Bi-enzyme cleavage with Nhe I and Sal I (New England BioLabs, Inc., Ipswich, MA, USA) was performed on the amplified fragments at $37^{\circ} \mathrm{C}$ for $3 \mathrm{~h}$, which were then ligated with purified fragments from the pELNS A recombinant lentivirus (Wuhan Genesil Biotechnology Co., Ltd., Wuhan, China) that had been bi-enzyme-digested, the recombinant was than enzyme-digested and sent for the further sequencing and confirmation.

Construction of lentiviral plasmid. According to the the method of a previous study (12), the alkaline lysis method was used prepare the target gene-constructed plasmids, pELNS-BMP2, pELNS-BMP4, pELNS-BMP6, pELNS-BMP7, pELNS-BMP9 and pELNS-Wnt3a, and the envelope protein plasmid $\mathrm{pLP} /$ vesicular stomatitis virus $\mathrm{G}$ glycoprotein (VSVG; Invitrogen; Thermo Fisher Scientific, Inc., Waltham, MA, USA), and the packaging protein plasmid pLP1 and pLP2 (Invitrogen; Thermo Fisher Scientific, Inc.). This vector system used enhanced green fluorescent protein (EGFP) as the reporter gene.

The $\mathrm{Ca}_{3}\left(\mathrm{PO}_{4}\right)_{2}$ coprecipitation method was used to transfect the 4 types of plasmid (pELNS target gene plasmid, $3 \mu \mathrm{g}$; pLP/VSVG plasmid, $2.8 \mu \mathrm{g}$; pLP1 plasmid, $4.2 \mu \mathrm{g}$; and pLP2 plasmid, $2 \mu \mathrm{g}$ ) into 293FT cells (American Type Culture Collection, Manassas, VA, USA), which were incubated with $10 \%$ fetal bovine serum (Sigma-Aldrich, St. Louis, MO, USA), at $37^{\circ} \mathrm{C}$ and $5 \% \mathrm{CO}_{2}$. Transduction was conducted using Lipofectamine 2000 transfection reagent (Gibco; Thermo Fisher Scientific, Inc.). Following, cotransfection for $72 \mathrm{~h}$, the supernatant was collected, filtered with $0.22 \mu \mathrm{m}$ filter and stored at $-80^{\circ} \mathrm{C}$. Counting of the number of green fluorescent protein (GFP)-positive cells was used to detect the viral titer, with the specific method described by Sugiyama et al (11).

Transfection of recombinant lentivirus into MMSCs MC3T3-E1. MC3T3-E1 MMSCs (American Type Culture Collection) were cultured in low-glucose Dulbecco's modified Eagle's complete medium (Gibco; Thermo Fisher Scientific, Inc.), supplemented with $10 \%$ fetal bovine serum at $37^{\circ} \mathrm{C}$ and $5 \% \mathrm{CO}_{2}$ for culture to the third or fourth passage. A preliminary experiment was performed to determine the optimal multiplicity of infection (MOI) of each virus when infecting MC3T3-E1 cells. MC3T3-E1 cells were infected according to the viral titer and best MOI for each virus using polybrene at a final concentration of $8 \mu \mathrm{g} / \mathrm{ml}$, and were observed and identified under a BX61 fluorescent microscope (Olympus Corporation, Tokyo, Japan) $72 \mathrm{~h}$ later. The single-gene transfection groups (6 groups) were as follows: pELNS-BMP2, pELNS-BMP4, pELNS-BMP6,
pELNS-BMP7, pELNS-BMP9 and pELNS-Wnt3a. The dual-gene cotransfection groups (8 groups) were as follows: T1, BMP2,4; T2, BMP4,7; T3, BMP2,9; T4, BMP7,9; T5, BMP2,7; T6, BMP4,9; T7, BMP2,6; and T8, BMP2 and Wnt3a.

Detection of runt related transcription factor 2 (Runx2) $m R N A$ by reverse transcription-quantitative $P C R(R T-q P C R)$. Runx 2 is a crucial gene for osteogenesis. The Runx 2 mRNA expression level in each group was detected. TRIzol (Invitrogen; Thermo Fisher Scientific, Inc.) was used to extract the total cellular RNA. The RT reaction with $2 \mu \mathrm{g}$ total RNA was performed using the PrimeScript First Strand cDNA Synthesis kit (Takara Biotechnology Co., Ltd., Dalian, China) according to the manufacturer's instructions. Glyceraldehyde 3-phosphate dehydrogenase (GAPDH) was used as the internal reference gene. The primers used in the PCR were as follows: Runx2, F 5'-ACAGAACCACAAGTGCGGTGC-3' and R 5'-ACTGTG GTTAGAGAGCACTCACTGA-3'; GAPDH, F 5'-AGGCGC TCACTGTTCTCTCCC-3' and R 5'-GCAAGGCTCGTAGAC GCGGTT-3'. The PCR cycling conditions were as follows: $95^{\circ} \mathrm{C}$ for $1 \mathrm{~min} ; 40$ cycles at $94^{\circ} \mathrm{C}$ for $5 \mathrm{sec}, 60^{\circ} \mathrm{C}$ for $20 \mathrm{sec}$ and $72^{\circ} \mathrm{C}$ for $10 \mathrm{sec}$; then final extension at $72^{\circ} \mathrm{C}$ for $5 \mathrm{~min}$. The relative mRNA levels were calculated using the $2^{-\Delta \Delta C q}$ method (13).

Expression level detection of bone $\gamma$-carboxyglutamate protein $(B G P)$ and alkaline phosphatase (ALP) by enzyme-linked immunosorbent assay (ELISA). After four generations of cell passage followed by transfection, the supernatant of each group was collected and the absorbance was measured using a microplate reader (R\&D Systems, Inc., Minneapolis, MN, USA) according to the specifications of the BGP ELISA kit (cat. no. 682556) and ALP ELISA kit (cat. no. 687242) (Antibody Research Corp., St. Charles, MO, USA). Based on the measurement results of standards, the contents of BGP and ALP in the culture supernatant were calculated.

Protein level detection of BMPs and Wnt3a in BMP2 and 7 cotransfected MC3T3-E1 cells by western blotting. The protein expression levels of BMPs and Wnt3a in BMP2and BMP7-cotransfected MC3T3-E1 cells were detected by western blotting according to a previously reported method (14). In brief, primary antibodies as goat polyclonal BMP2, BMP4, BMP6 and BMP7 antibodies, and rabbit polyclonal BMP9, Wnt3a and $\beta$-actin antibodies were purchased from Santa Cruz Biotechnology, Inc. (Dallas, TX, USA). The target stripes were then observed.

Statistical analysis. All data are expressed as the mean \pm standard deviation $(\mathrm{x} \pm \mathrm{s})$. SPSS statistical software (version 17.0; SPSS, Inc., Chicago, IL, USA) was used to perform statistical analysis. The multiple-sample comparisons were performed using one way analysis of variance and the intergroup comparison using the least significant difference test.

\section{Results}

Identification of target gene-expressed plasmid. All the target gene-expressed vectors were digested by Nhe I and Sal I. The 
Table I. Primer sequences of BMPs and Wnt3a.

Primer

Sequence

Fragment

M-BMP2-P

F 5'-CTAGCTAGCATGGTGGCCGGGACCCGCTGTCTTC-3'

size (bp)

$\begin{array}{cc}\text { M-BMP4-P 5'-ACGCGTCGACCTAACGACACCCGCAGCCCTCCAC-3' } \\ \text { F 5'-CTAGCTAGCATGATTCCTGGTAACCGAATGCTG-3' } \\ \text { R 5'-ACGCGTCGACTCAGCGGCATCCACACCCCTCTAC-3' } \\ \text { M-BMP6-P } \\ \text { F 5'-CTAGCTAGCATGCCCGGGCTGGGGCGGAG-3' } \\ \text { M-BMP7-P } & \text { R 5'-ACGCGTCGACTTAATGGCAACCACAAGCTCTCACGACC-3' } \\ & \text { R 5'-ACGCGAGCTAGCATGCACGTGCGCTCGCTGCG-3' } \\ \text { M-BMP9-P } & \text { F 5'-CTAGCTAGCATGTCCCCTGGGGCCTTCCG-3' } \\ & \text { R 5'-ACGCGTCGACCTACCTACACCCACACTCAGCC-3' } \\ \text { M-Wnt3a-P } & \text { F 5'-CTAGCTAGCATGGCTCCTCTCGGATACCTCTTAG-3' } \\ & \text { R 5'-ACGCGTCGACCTACTTGCAGGTGTGCACGTCATAG-3' }\end{array}$

BMP, bone morphogenic protein; F, forward; R, reverse.

electrophoresis separation resulted in two bands; one was the linearized plasmid DNA, while the other was the target gene band. The size of each target gene fragment was consistent with the design (Fig. 1), and the sequencing results were consistent with those in Genbank (www.ncbi.nlm.nih.gov/genbank), indicating that the expression vectors were successfully constructed.

Transfection of single-gene recombinant lentivirus into MC3T3-E1 cells. Fluorescence microscopy demonstrated that the infected cells exhibited green fluorescence, indicating the recombinant lentivirus had been successfully infected into MC3T3-E1 cells (Fig. 2).

Detection of Runx 2 mRNA by RT-qPCR. In the single transfection cells, the results of RT-qPCR demonstrated that the relative expression level of Runx 2 mRNA in the BMP2 group was the highest, sequentially followed by the BMP4 group, BMP9 group, BMP7 group, Wnt3a group and BMP6 group (Fig. 3A), and the differences between the test groups and the control were statistically significant as indicated in Fig. 3A $(\mathrm{P}<0.05)$.

In the bi-gene combined transfection cells, the results demonstrated that the expression level of Runx2 mRNA was highest in the T5 (BMP2,7) group, followed by T3 (BMP2,9) group, T8 (BMP2 and Wnt3a) group, T1 $(\mathrm{BMP} 2,4)$ group, T2 $(\mathrm{BMP} 4,7)$ group, T6 (BMP4,9) group, T4 (BMP7,9) group and T7 (BMP2,6) group (Fig. 3B), and the differences between the test groups (excluding T7) and the control were statistically significant as indicated in Fig. 3B $(\mathrm{P}<0.05)$.

$B G P$ and ALP ELISA. The results demonstrated that the expression level of BGP in the T5 (BMP2,7) group was the highest, sequentially followed by the T3 group, T8 group, T4 group, T2 group, T6 group, T1 group and T7 group (Table II). The expression level of ALP in T5 (BMP2,7) group was the highest, followed by the T3 group, T1 group, T8 group,

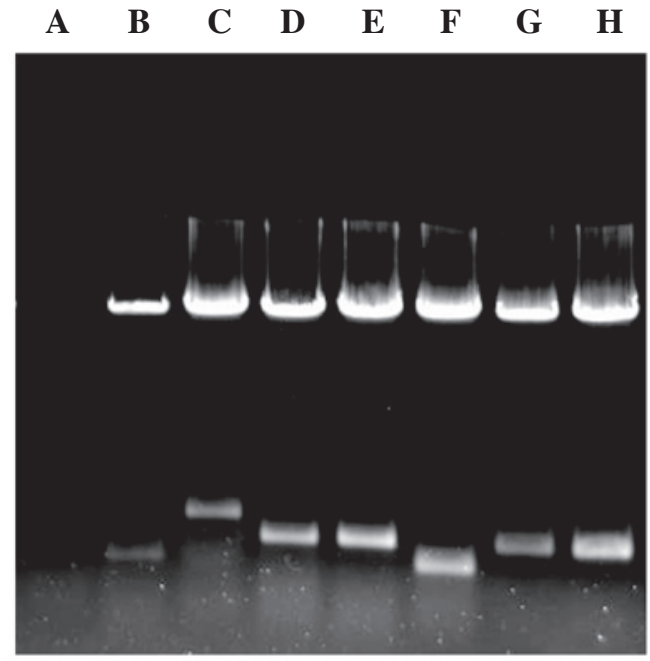

Figure 1. Agarose gel electrophoresis images of expression plasmids following digestion. (A) Original single-enzyme digested linear pELNS plasmid; (B) original bi-enzyme digested linear pELNS plasmid, producing $1 \mathrm{~kb}$ rtTA; (C) double digestion of pELNS-BMP6, producing $1.5 \mathrm{~kb}$ BMP6; (D) double digestion of pELNS-BMP7, producing $1.3 \mathrm{~kb}$ BMP7; (E) double digestion of pELNS-BMP9, producing 1.3 kb BMP9; (F) double digestion of pELNS-Wnt3a, producing $1 \mathrm{~kb}$ Wnt3a; (G) double digestion of pELNS-BMP4, producing 1.2 kb BMP4; $(\mathrm{H})$ double digestion of pELNS-BMP2, producing 1.2 kb BMP2. BMP, bone morphogenic protein.

T2 group, T4 group, T6 group and T7 group (Table II), the differences between values in the $\mathrm{T} 1, \mathrm{~T} 2, \mathrm{~T} 3, \mathrm{~T} 4, \mathrm{~T} 5, \mathrm{~T} 6$, $\mathrm{T} 7$ and T8 groups with the control group were statistically significant $(\mathrm{P}<0.05)$.

Fluorescence detection. The T5 (BMP2,7) group cells exhibited green fluorescence, indicating the lentivirus was successfully infected into MC3T3-E1 cells (Fig. 4).

Western blot analysis. The results demonstrated that BMP2 and BMP7 (T5 group) cotransfected MC3T3-E1 


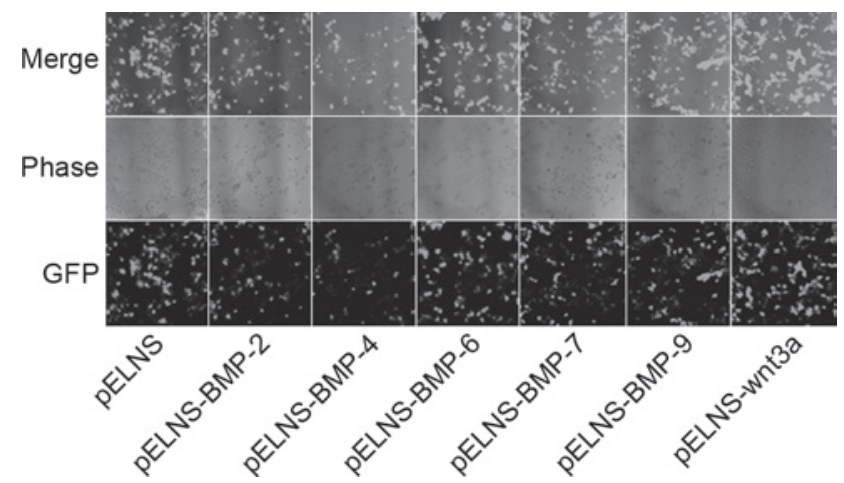

Figure 2. Fluorescence microscopy of lentivirus-transfected MC3T3-E1 cells. GFP, green fluorescent protein; BMP, bone morphogenic protein.

$\mathbf{A}$

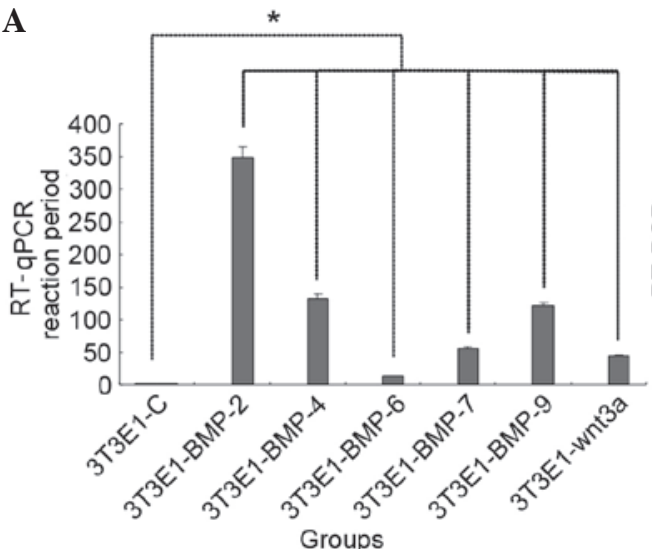

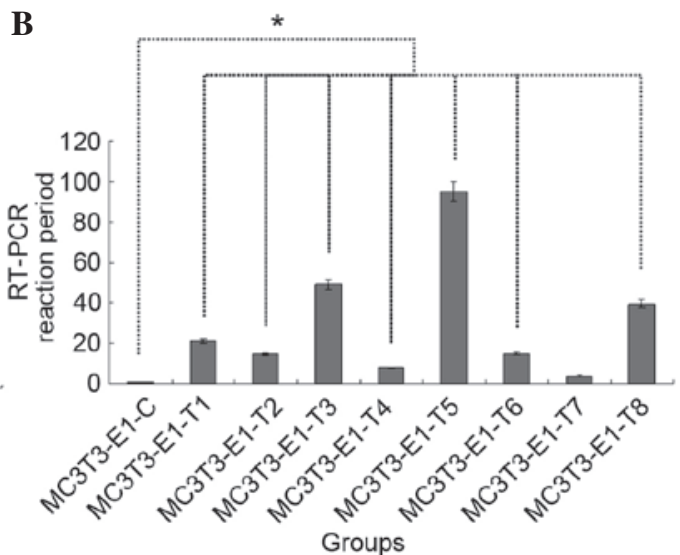

Figure 3. Expression level of runt related transcription factor 2 mRNA in MC3T3-E1 cells. (A) Single transfection; (B) bi-gene combined transfection. "P<0.05. RT-qPCR; reverse transcription-quantitative polymerase chain reaction; $\mathrm{C}$, control; BMP, bone morphogenic protein.

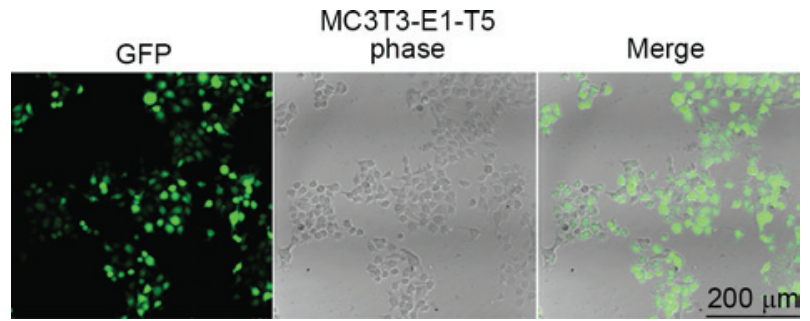

Figure 4. Fluorescence microscopy of BMP2 and BMP7 co-transfected MC3T3-E1 cells. GFP, green fluorescent protein; BMP, bone morphogenic protein.

cells exhibited higher expression levels of BMP-2, BMP-4, BMP-6, BMP-7, BMP-9 and Wnt3a protein compared with untransfected cells (Fig. 5).

\section{Discussion}

Genetically modified stem cells. The crucial element and research focus of bone tissue engineering technology has been how to promote cell proliferation and osteogenic differentiation. The transplantation therapy of in vitro gene-modified stem cells was a combination of cell therapy and gene therapy, compared with adult cells, the stem cells exhibited a higher proliferation ability and increased survival time. Furthermore, a previous study demonstrated that gene-modified MSCs exhibited long-term expression of exogenous genes, and

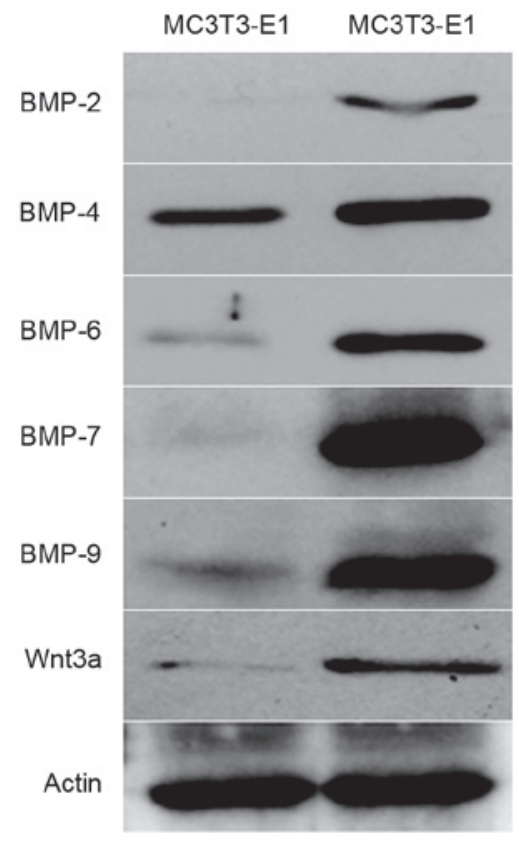

Figure 5. Expression levels of BMPs and Wnt3a protein in BMP2 and BMP7 co-transfected MC3T3-E1 cells. BMP, bone morphogenic protein.

maintained this ability following transplantation into the body (15). Thus, gene-modified MSCs may overcome the loss of its therapeutic effects. Additionally, they could be used as 
Table II. Absorbance values of BGP and ALP in 8 groups of cotransfected MC3T3-E1 cells by enzyme-linked immunosorbent assay.

\begin{tabular}{|c|c|c|c|c|}
\hline \multirow[b]{2}{*}{ Group } & \multicolumn{2}{|c|}{ BGP } & \multicolumn{2}{|c|}{ ALP } \\
\hline & 1 st & 2nd & 1 st & 2nd \\
\hline MSCs control & $0.026 \pm 0.005$ & $0.035 \pm 0.006$ & $0.004 \pm 0.004$ & $0.037 \pm 0.003$ \\
\hline Positive control & $1.05 \pm 0.02$ & $1.72 \pm 0.04$ & $1.02 \pm 0.36$ & $1.64 \pm 0.38$ \\
\hline $\mathrm{T} 1$ & $0.21 \pm 0.01^{\mathrm{a}}$ & $0.34 \pm 0.01^{\mathrm{a}}$ & $0.44 \pm 0.04^{\mathrm{a}}$ & $0.63 \pm 0.08^{\mathrm{a}}$ \\
\hline $\mathrm{T} 2$ & $0.25 \pm 0.003^{\mathrm{a}}$ & $0.42 \pm 0.01^{\mathrm{a}}$ & $0.33 \pm 0.09^{\mathrm{a}}$ & $0.59 \pm 0.04^{\mathrm{a}}$ \\
\hline $\mathrm{T} 3$ & $0.43 \pm 0.01^{\mathrm{a}}$ & $0.71 \pm 0.02^{\mathrm{a}}$ & $0.61 \pm 0.13^{\mathrm{a}}$ & $0.78 \pm 0.09^{\mathrm{a}}$ \\
\hline $\mathrm{T} 4$ & $0.28 \pm 0.01^{\mathrm{a}}$ & $0.46 \pm 0.01^{\mathrm{a}}$ & $0.37 \pm 0.07^{\mathrm{a}}$ & $0.49 \pm 0.05^{\mathrm{a}}$ \\
\hline T5 & $0.60 \pm 0.01^{\mathrm{a}}$ & $0.97 \pm 0.02^{\mathrm{a}}$ & $0.62 \pm 0.17^{\mathrm{a}}$ & $0.90 \pm 0.17^{\mathrm{a}}$ \\
\hline T6 & $0.25 \pm 0.01^{\mathrm{a}}$ & $0.41 \pm 0.01^{\mathrm{a}}$ & $0.33 \pm 0.06^{\mathrm{a}}$ & $0.44 \pm 0.06^{\mathrm{a}}$ \\
\hline $\mathrm{T} 7$ & $0.23 \pm 0.004^{\mathrm{a}}$ & $0.18 \pm 0.006^{\mathrm{a}}$ & $0.14 \pm 0.05^{\mathrm{a}}$ & $0.16 \pm 0.04^{\mathrm{a}}$ \\
\hline $\mathrm{T} 8$ & $0.31 \pm 0.007^{\mathrm{a}}$ & $0.50 \pm 0.006^{\mathrm{a}}$ & $0.42 \pm 0.09^{\mathrm{a}}$ & $0.56 \pm 0.17^{\mathrm{a}}$ \\
\hline
\end{tabular}

${ }^{\text {a }}<<0.05$ vs. MSCs control group. BGP, bone $\gamma$-carboxyglutamate protein; ALP, alkaline phosphatase; MSCs, mesenchymal stem cells.

a vector to express osteogenic differentiation regulators for gene therapy, thus compared with simple cell transplantation or gene therapy, may provide improved treatment effects (16).

Selection of viral vectors. The ideal carrier for gene therapy should allow the efficient transduction and long-term stable expression of the transgene, and result in minimal side effects. The most commonly used viral vectors are predominantly retrovirus, adenovirus, adeno-associated virus and lentivirus (17-20).

Lentivirus is a subfamily of retrovirus. Compared with adenoviruses, adeno-associated viruses and retroviruses, the greatest advantages of using a lentivirus are the longer gene expression time, reduced host immune responses, lower cytotoxicity and easier use. In a previous study, a lentivirus-mediated transgene remained expressed at 6 months after transduction, and the concomitant inflammation caused was lower compared with that of adenoviruses (21). Additionally, lentiviruses have strong packaging capacities, which can hold up to 8-10 kb of exogenous target gene fragments, while unlikely to cause insertional mutagenesis. Thus, lentiviruses have broad prospects for gene therapy (22). The third-generation lentiviral vector system induces genes to be inserted into the lentiviral vector, thus it artificially controls the expression of transferred genes, and retains its inactivated properties, thus the system is also known as the regulable lentiviral vector (23).

In order to improve the biological safety of viral vectors, the current study used improved four-plasmid packaging system, thus, separated the encoding genes of packaging plasmid, and the packaging system was composed of packaging plasmid pLP1 (encoding gag/Pol gene) and pLP2 (encoding Rev gene), envelope protein plasmid pLP/VSVG and vector plasmid pELNS. This vector system only retained the gag, Pol and Rev genes of human immunodeficiency virus-1, and there was no autoploidy within the four plasmids of this four-plasmid cotransfection system. This ensured the reduced possibility of replication competent virus generation. This vector system replaced the cytomegalovirus (CMV) internal promoter with the eukaryotic elongation factor- $1 \alpha(\mathrm{EF}-1 \alpha)$ promoter, based on the pRRL-SIN-CMV-EGFP-WPRE plasmid, and the promoter effects EF-1 $\alpha$ were stronger. This carrier system used EGFP as the reporter gene, thus, the advantages were: i) EGFP does not interfere with cell growth and functions, the EGFP-carrying fusion gene protein not exhibited the EGFP green fluorescence, it also maintained the physiological functions of target proteins and exhibited no cytotoxicity (24), and allowed detection of the gene expression levels in living cells; ii) the observation was simple, EGFP was used as a reporter gene that could be directly observed in living cells with no need of a substrate and internal control, when the EGFP gene was transfected into eukaryotic cells and expressed, it could easily observe the green fluorescent distribution of EGFP fusion protein inside the living cells to analyze the distribution and localization of unknown gene-expressed proteins within the cells; and iii) the EGFP-labeling method exhibited higher sensitivity and resolution than immunohistochemistry and conventional labeling methods. Thus, as a very promising marker, EGFP provided great convenience for the study of gene function and expression regulation. The expression levels of EGFP green fluorescence confirmed the effects of plasmid transfection, and the conditions of packaging, infection and exogenous gene expression of the recombinant virus inside cells. The present study demonstrated that the third-generation lentiviral vector pELNS successfully introduced BMP2, BMP4, BMP6, BMP7, BMP9 and Wnt3a into MMSCs MC3T3-E1, and the expression was stable $(25,26)$.

Osteogenic differentiation-inducing factor. Different members of BMP family have different distributions and osteogenic activity. Studies have previously suggested that BMP2 predominantly stimulates recruitment and differentiation of the undifferentiated mesenchymal cells and bone cells, thus participating the bone reconstruction. In 1996, Iwasaki demonstrated that the specific receptors for BMP2 are expressed on the surface of bone cells and on the surface of all non-hematopoietic stem cells (27). 
A previous study has demonstrated that BMP4 inhibited the proliferation of lung fibroblasts through the classic Smad I and c-Jun-N-terminal kinase pathway, and promoted their differentiation into muscle cells (28). In vitro experiments demonstrated that BMP4 promoted osteoblastic differentiation and exerted a catalytic effect on bone collagen production (29). BMP6 was demonstrated to be important at an earlier stage of bone formation and induced the differentiation of precursor cells of chondrocytes, promoted the formation of cartilage and osteoblastic phenotype, thus, involved in cartilage and bone formation. Compared with other BMP family members, BMP6 generated stronger osteoinductive effects on intracartilage and intramembranous ossification (30). BMP7 have been demonstrated to bind with BMP type I and II receptors stimulating Smad5 signaling to the nucleus and influencing the gene transcription, thus, promoting the formation of bone and cartilage, and repairing defects (31). BMP9 (also termed growth differentiation factor 2) was previously demonstrated to be a potential synergic promoting factor involved in the proliferation and colony formation of hematopoietic stem progenitor cells, and associated with the differentiation maintenance of cholinergic nerve cells in the central nervous system. BMP9 was also demonstrated to be involved in regulating glucose metabolism and a variety of other cellular functions. Among numerous biological effects of BMP9, its effect on bone and cartilage formation remains the most important (32).

The Wnt family, and its various homologs, are secreted glycoproteins. Their functions vary with different tissues or developmental stages. The Wnt genes are proto-oncogenes, and the Wnt signaling pathway involves the transduction of a signal from the cell surface to the nucleus via a secreted signaling protein, a transmembrane receptor protein and various complex intracellular proteins (33).

Runx 2 is part of the Runx-domain gene family, and among various osteogenic differentiation regulatory factors, Runx2 has been considered to be the master regulator of osteoblast differentiation. Runx 2 is expressed within the mesenchyme of mineralized tissues, including bones, and in the early development stage of epithelial tissues (34). The present study detected the expression of Runx 2 mRNA levels, thus, evaluated its involvement in osteogenic differentiation.

The current study successfully constructed the recombinant lentiviruses, pELNS-BMP-2, pELNS-BMP-4, pELNS-BMP-6, pELNS-BMP-7, pELNS-BMP-9 and pELNS-Wnt3a, and transfected them into MC3T3-E1 cells. By detecting the expression level changes of Runx 2 mRNA, it was demonstrated that the osteogenic effects of BMP2 were the strongest, and those of BMP6 were the weakest compared with controls. The effect of combined transfection of BMP2 with BMP4, BMP6, BMP7, BMP9 and Wnt3a, and the combination of BMP4 with BMP7, BMP4 with BMP9, and BMP7 with BMP9, were determined in MC3T3-E1 cells. The results demonstrated that the osteogenic effects of BMP7 with BMP2 were the strongest. ELISA results demonstrated that the expression level of BGP in the T5 (BMP2,7) group was the highest, followed by the T3, T8, T4, T2, T6, T1 and T7 groups. The expression level of ALP in the T5 (BMP2,7) group was the highest, sequentially followed by the T3, T1, T8, T2, T4, T6 and T7 groups. BMP2 and BMP7 have previously been considered to be the most effective and promising clinical osteogenic factors, with recombinant human BMP2 and BMP7 used as bone regeneration therapies for bone delayed union, bone nonunion, bone defects and spinal fusion surgery (35). However recent research and clinical practice has indicated that the use of recombinant BMP2 and BMP7 protein could not solve the medical problem of bone regeneration (36). Thus, how to improve the osteoinductive abilities of cells and to provide efficient, inexpensive and convenient bone regeneration materials has become a common topic of concern, and urgently required in the clinical. BMP2 combined with BMP7 may improve the osteoinductive abilities. The current study established that cotransfection with the BMP2 and BMP7 lentiviruses effectively induced the osteogenic differentiation of MC3T3-E1 cells, thus laying the foundation for future in vivo studies. In conclusion, the third-generation lentiviral vectors effectively improved the osteogenic efficiencies of MC3T3-E1 cells, which provided an important theoretical basis and therapeutic strategy for bone reconstruction and tissue engineering.

\section{References}

1. Grässel S and Lorenz J: Tissue-engineering strategies to repair chondral and osteochondral tissue in osteoarthritis: Use of mesenchymal stem cells. Curr Rheumatol Rep 16: 452, 2014.

2. Ochi M, Nakasa T, Kamei G, Usman MA and Mahmoud E: Regenerative medicine in orthopedics using cells, scaffold and microRNA. J Orthop Sci 19: 521-528, 2014.

3. Bayrak ES, Mehdizadeh H, Akar B, Somo SI, Brey EM and Cinar A: Agent-based modeling of osteogenic differentiation of mesenchymal stem cells in porous biomaterials. Conf Proc IEEE Eng Med Biol Soc 2014: 2924-2927, 2014.

4. Oryan A, Alidadi S, Moshiri A and Maffulli N: Bone regenerative medicine: Classic options, novel strategies and future directions. J Orthop Surg Res 9: 18, 2014.

5. Kwon TK, Song JM, Kim IR, Park BS, Kim CH, Cheong IK and Shin SH: Effect of recombinant human bone morphogenetic protein-2 on bisphosphonate-treated osteoblasts. J Korean Assoc Oral Maxillofac Surg 40: 291-296, 2014.

6. Yan F, Luo S, Jiao Y, Deng Y, Du X, Huang R, Wang Q and Chen W: Molecular characterization of the BMP7 gene and its potential role in shell formation in Pinctada martensii. Int $\mathrm{J}$ Mol Sci 15: 21215-21228, 2014.

7. Wang RN, Green J, Wang Z, Deng Y, Qiao M, Peabody M, Zhang Q, Ye J, Yan Z, Denduluri S, et al: Bone morphogenetic protein (BMP) signaling in development and human diseases. Genes Dis 1: 87-105, 2014.

8. Wang Y, Li YP, Paulson C, Shao JZ, Zhang X, Wu M and Chen W: Wnt and the Wnt signaling pathway in bone development and disease. Front Biosci (Landmark Ed) 19: 379-407, 2014.

9. Zahoor M, Cha PH, Min do S and Choi KY: Indirubin-3'-oxime reverses bone loss in ovariectomized and hindlimb-unloaded mice via activation of the Wnt/ $\beta$-catenin signaling. J Bone Miner Res 29: 1196-1205, 2014.

10. Heilbronn R and Weger S: Viral vectors for gene transfer: Current status of gene therapeutics. Handb Exp Pharmacol 197: $143-170,2010$.

11. Sugiyama O, An DS, Kung SP, Feeley BT, Gamradt S, Liu NQ, Chen IS and Lieberman JR: Lentivirus-mediated gene transfer induces long-term transgene expression of BMP-2 in vitro and new bone formation in vivo. Mol Ther 11: 390-398, 2005.

12. Benskey MJ and Manfredsson FP: Lentivirus production and purification. Methods Mol Biol 1382: 107-114, 2016.

13. Livak KJ and Schmittgen TD: Analysis of relative gene expression data using real-time quantitative PCR and the 2(-Delta Delta C(T)) Method. Methods 25: 402-408, 2001.

14. Krause C, Korchynskyi O, de Rooij K, Weidauer SE, de Gorter DJ, van Bezooijen RL, Hatsell S, Economides AN, Mueller TD, Löwik CW and ten Dijke P: Distinct modes of inhibition by sclerostin on bone morphogenetic protein and Wnt signaling pathways. J Biol Chem 285: 41614-41626, 2010. 
15. Zhou S, Zilberman Y, Wassermann K, Bain SD, Sadovsky Y and Gazit D: Estrogen modulates estrogen receptor alpha and beta expression, osteogenic activity, and apoptosis in mesenchymal stem cells (MSCs) of osteoporotic mice. J Cell Biochem Suppl 36 : 144-155, 2001.

16. Hernigou P, Flouzat-Lachaniette $\mathrm{CH}$, Delambre J, Poignard A, Allain J, Chevallier N and Rouard H: Osteonecrosis repair with bone marrow cell therapies: State of the clinical art. Bone 70 : 102-109, 2015

17. Virk MS, Conduah A, Park SH, Liu N, Sugiyama O, Cuomo A Kang $\mathrm{C}$ and Lieberman JR: Influence of short-term adenoviral vector and prolonged lentiviral vector mediated bone morphogenetic protein-2 expression on the quality of bone repair in a rat femoral defect model. Bone 42: 921-931, 2008.

18. Kotterman MA and Schaffer DV: Engineering adeno-associated viruses for clinical gene therapy. Nat Rev Genet 15: 445-451, 2014.

19. Deichmann A and Schmidt M: Biosafety considerations using gamma-retroviral vectors in gene therapy. Curr Gene Ther 13 469-477, 2013

20. Maier P, von Kalle C and Laufs S: Retroviral vectors for gene therapy. Future Microbiol 5: 1507-1523, 2010.

21. Pauwels K, Gijsbers R, Toelen J, Schambach A, Willard-Gallo K, Verheust C, Debyser Z and Herman P: State-of-the-art lentiviral vectors for research use: Risk assessment and biosafety recommendations. Curr Gene Ther 9: 459-474, 2009.

22. Rothe M, Modlich U and Schambach A: Biosafety challenges for use of lentiviral vectors in gene therapy. Curr Gene Ther 13 453-468, 2013

23. Dull T, Zufferey R, Kelly M, Mandel RJ, Nguyen M, Trono D and Naldini L: A third-generation lentivirus vector with a conditional packaging system. J Virol 72: 8463-8471, 1998.

24. Miller F, Hinze U, Chichkov B, Leibold W, Lenarz T, Paasche G: Validation of eGFP fluorescence intensity for testing in vitro cytotoxicity according to ISO 10993-5. J Biomed Mater Res B Appl Biomater: Dec 24, 2015 (Epub ahead of print).

25. Witting SR, Vallanda P and Gamble AL: Characterization of a third generation lentiviral vector pseudotyped with Nipah virus envelope proteins for endothelial cell transduction. Gene Ther 20: 997-1005, 2013
26. Mátrai J, Chuah MK and VandenDriessche T: Recent advances in lentiviral vector development and applications. Mol Ther 18: 477-490, 2010.

27. Iwasaki S, Hattori A, Sato M, Tsujimoto $M$ and Kohno M Characterization of the bone morphogenetic protein-2 as a neurotrophic factor. Induction of neuronal differentiation of PC12 cells in the absence of mitogen-activated protein kinase activation. J Biol Chem 271: 17360-17365, 1996.

28. Usas A, Ho AM, Cooper GM, Olshanski A, Peng H and Huard J: Bone regeneration mediated by BMP4-expressing muscle-derived stem cells is affected by delivery system. Tissue Eng Part A 15: 285-293, 2009.

29. Jeffery TK, Upton PD, Trembath RC and Morrell NW: BMP4 inhibits proliferation and promotes myocyte differentiation of lung fibroblasts via Smad1 and JNK pathways. Am J Physiol Lung Cell Mol Physiol 288: L370-L378, 2005.

30. Gitelman SE, Kirk M, Ye JQ, Filvaroff EH, Kahn AJ and Derynck R: Vgr-1/BMP-6 induces osteoblastic differentiation of pluripotential mesenchymal cells. Cell Growth Differ 6: 827-836, 1995.

31. Desmyter S, Goubau Y, Benahmed N, de Wever A and Verdonk R: The role of bone morphogenetic protein-7 (Osteogenic Protein-1) in the treatment of tibial fracture non-unions. An overview of the use in Belgium. Acta Orthop Belg 74: 534-537, 2008.

32. Bibbo C, Nelson J, Ehrlich D and Rougeux B: Bone morphogenetic proteins: Indications and uses. Clin Podiatr Med Surg 32: 35-43, 2015.

33. Zhou S, Mizuno S and Glowacki J: Wnt pathway regulation by demineralized bone is approximated by both BMP-2 and TGF- $\beta 1$ signaling. J Orthop Res 31: 554-560, 2013.

34. Wagner ER, Zhu G, Zhang BQ, Luo Q, Shi Q, Huang E, Gao Y, Gao JL, Kim SH, Rastegar F, et al: The therapeutic potential of the Wnt signaling pathway in bone disorders. Curr Mol Pharmacol 4: 14-25, 2011.

35. Conway JD, Shabtai L, Bauernschub A and Specht SC: BMP-7 versus BMP-2 for the treatment of long bone nonunion. Orthopedics 37: e1049-e1057, 2014.

36. Cirano FR, Togashi AY, Marques MM, Pustiglioni FE and Lima LA: Role of rhBMP-2 and rhBMP-7 in the metabolism and differentiation of osteoblast-like cells cultured on chemically modified titanium surfaces. J Oral Implantol 40: 655-659, 2014. 